\title{
EXISTENCE AND UNIQUENESS OF SOLUTIONS TO A FIRST-ORDER DIFFERENTIAL EQUATION VIA FIXED POINT THEOREM IN ORTHOGONAL METRIC SPACE
}

\author{
Madjid Eshaghi Gordji and Hasti Habibi
}

\begin{abstract}
In this paper we provide new and simple proofs for the classical existence and uniqueness theorems of solutions to the first-order differential equation using the fixed point theorem in an orthogonal metric space.

Keywords: Fixed point; Differential equation; Existence; Uniqueness; Solution; Orthogonal set.
\end{abstract}

\section{Introduction}

Let us consider the differential equation

$$
\dot{x}(t)=v(t, x), \quad x\left(t_{0}\right)=x_{0},
$$

where $t \in \mathbb{R}, x \in \mathbb{R}^{n}$ and $v(t, x)$ is defined and differentiable (of class $C^{r}, r \geq 1$ ) in a domain $U$ of $\mathbb{R} \times \mathbb{R}^{n}$.

The solution to this equation will be a function $\phi: \mathbb{R} \rightarrow \mathbb{R}^{n}$ where

$$
\dot{\phi}(t)=v(t, \phi(t)), \quad \phi\left(t_{0}\right)=x_{0} .
$$

The existence and uniqueness of solutions to first-order differential equations with given initial conditions are some of the most fundamental results of ordinary differential equations. This is stated in the two following theorems.

Theorem 1.1. [8] (The Existence Theorem) Suppose the right-hand side $v$ of the differential equation $\dot{x}(t)=v(t, x)$ is continuously differentiable in a neighborhood of the point $\left(t_{0}, x_{0}\right) \in \mathbb{R} \times \mathbb{R}^{n}$. Then there exists a neighborhood of the point $t_{0}$ such that a solution of the differential equation is defined in this neighborhood with the initial condition $\phi\left(t_{0}\right)=x_{0}$, where $x$ is any point sufficiently close to $x_{0}$. Moreover, this solution depends continuously on the initial point $x$.

Received January 17, 2017; accepted March 12, 2018

2010 Mathematics Subject Classification. Primary 47H10; Secondary 54H25 
Theorem 1.2. [8](The Uniqueness Theorem) Given the above conditions, there is only one possible solution for any given initial point, in the sense that all possible solutions are equal in the neighborhood under consideration.

Previous studies have provided proofs of Theorems 1.1 and 1.2 using the concepts of Banach contraction principle [1, 7, 8], [12] and [16, 15].

Recently, M. Eshaghi et.al. [13] introduced the concept of orthogonal sets. A real extension of Banach contraction principle in orthogonal metric space has been considered in [13] (see also $[9,10,19])$. In this paper, we are interested in obtaining new and simple proofs for Theorems 1.1 and 1.2 which guarantee existence and uniqueness of the solution for any equation of the form (1.1).

This paper is organized as follows: In section 2, we state some definitions and theorems which are needed to prove the main results. Also, we recall under what conditions will any mapping on an orthogonal metric space have a unique fixed point. In section 3, we consider new concepts of tangent space to an orthogonal metric space and derivative of mapping at a point in an orthogonal metric space. This section provides a priori bound for the solution. In this section, we make use of the standard tools of the fixed point theory in orthogonal metric spaces to obtain new and simple proofs for existence and uniqueness theorems of solutions for the differential equation (1.1).

\section{Preliminary definitions}

First, we begin with the following definition which can be considered as the main definition of [13].

Definition 2.1. [13] Let $M \neq \phi$ and $\perp \subseteq M \times M$ be a binary relation. If $\perp$ satisfies the following condition

$$
\exists x_{0} ;\left(\left(\forall y ; y \perp x_{0}\right) \text { or }\left(\forall y ; x_{0} \perp y\right)\right),
$$

it is called an orthogonal set (briefly $\mathrm{O}$-set). We denote this O-set by $(M, \perp)$ (see also $[9,10,19])$.

We now give some examples of orthogonal sets.

Example 2.1. Let $M=[2, \infty)$, we define $x \perp y$ if $x \leq y$, then by putting $x_{0}=2,(M, \perp)$ is an O-set.

In the following example, we can see that $x_{0}$ is not necessarily unique.

Example 2.2. Suppose $\mathcal{M}(n)$ is the set of all $n \times n$ matrices and $Q$ is a positive definite matrix. Define the relation $\perp$ on $\mathcal{M}(n)$ by

$$
A \perp B \Longleftrightarrow \exists X \in \mathcal{M}(n) ; \quad A X=B .
$$

It is easy to see that $I \perp B, B \perp 0$ and $Q^{\frac{1}{2}} \perp B$ for all $B \in \mathcal{M}(n)$. 
Now, we turn our consideration to the definition of orthogonal sequence.

Definition 2.2. $[13]$ Let $(M, \perp)$ be an O-set. A sequence $\left\{x_{n}\right\}_{n \in \mathbb{N}}$ is called orthogonal sequence (briefly $\mathrm{O}$-sequence) if

$$
\left(\forall n ; x_{n} \perp x_{n+1}\right) \text { or }\left(\forall n ; x_{n+1} \perp x_{n}\right) \text {. }
$$

(see also $[9,10,19])$.

Let $(M, \rho, \perp)$ be an orthogonal metric space $((M, \perp)$ is an O-set and $(M, \rho)$ is a metric space). We consider the notion of $\mathrm{O}$-complete orthogonal metric space.

Definition 2.3. [13] $M$ is orthogonally complete (briefly O-complete) if every Cauchy O-sequence is convergent (see also $[9,10,19]$ ).

Definition 2.4. Let $(M, \rho, \perp$ ) be an orthogonal metric space and $0<\lambda<1$ (see $[13])$.

1. A mapping $f: M \rightarrow M$ is said to be orthogonal contraction ( $\perp-$ contraction) with Lipchitz constant $\lambda$ if

$$
\rho(f x, f y) \leq \lambda \rho(x, y) \quad \text { if } x \perp y
$$

2. A mapping $f: M \rightarrow M$ is called orthogonality-preserving ( $\perp$-preserving) if $f(x) \perp f(y)$ if $x \perp y$.

3. A mapping $f: M \rightarrow M$ is continuous orthogonal ( $\perp$-continuous) in $a \in M$ if for each O-sequence $\left\{a_{n}\right\}_{n \in \mathbb{N}}$ in $M$ if $a_{n} \rightarrow a$, then $f\left(a_{n}\right) \rightarrow f(a)$. Also $f$ is $\perp$-continuous on $M$ if $f$ is $\perp$-continuous in each $a \in M$.

(see also $[9,10,19])$.

Example 2.3. Let $M=[0,1)$ and let the metric on $M$ be the Euclidian metric. Define $x \perp y$ if $x y \in\{x, y\} . M$ is not complete but it is O-complete. Let $x \perp y$ and $x y=x$. If $\left\{x_{k}\right\}$ is an arbitrary Cauchy $\mathrm{O}$-sequence in $M$, then there exists a subsequence $\left\{x_{k_{n}}\right\}$ of $\left\{x_{k}\right\}$ for which $x_{k_{n}}=0$ for all $n$. It follows that $\left\{x_{k_{n}}\right\}$ converges to a $x \in M$. On the other hand, we know that every Cauchy sequence with a convergent subsequence is convergent. It follows that $\left\{x_{k}\right\}$ is convergent.

Let $f: M \rightarrow M$ be a mapping defined by $f(x)=\frac{x}{2}$ if $x \in \mathbb{Q} \cap M$ and $f(x)=0$ if $x \in \mathbb{Q}^{c} \cap M$.

We have the following cases:

case 1) $x=0$ and $y \in \mathbb{Q} \cap M$. Then $f(x)=0$ and $f(y)=\frac{y}{2}$.

case 2) $x=0$ and $y \in \mathbb{Q}^{c} \cap M$. Then $f(x)=f(y)=0$.

This implies that $f(x) f(y)=f(x)$. Hence $f$ is $\perp$-preserving. Also, this implies that $|f(x)-f(y)| \leq \frac{1}{2}|x-y|$. Hence $f$ is $\perp-$ contraction. But $f$ is not a contraction. To see this, for each $\lambda<1,\left|f\left(\frac{1}{2}\right)-f\left(\frac{\sqrt{3}}{4}\right)\right|>\lambda\left|\frac{1}{2}-\frac{\sqrt{3}}{4}\right|$. 
If $\left\{x_{n}\right\}$ is an arbitrary O-sequence in $M$ such that $\left\{x_{n}\right\}$ converges to $x \in M$. Since $f$ is $\perp$-contraction, for each $n \in \mathbb{N}$ we have

$$
\left|f\left(x_{n}\right)-f(x)\right| \leq \frac{1}{2}\left|x_{n}-x\right| .
$$

As $n$ goes to infinity, $f$ is $\perp$-continuous. But it can be easily seen that $f$ is not continuous.

We can now state the main theoretical result of [13]. Sufficient conditions under which any mapping on an orthogonal metric space will have a unique fixed point are given in the theorem.

Theorem 2.1. Let $(M, \rho, \perp$ ) be an O-complete metric space (not necessarily complete metric space) and $0<\lambda<1$. Let $f: M \rightarrow M$ be $\perp$-continuous, $\perp$-contraction (with Lipschitz constant $\lambda$ ) and $\perp$-preserving, then $f$ has a unique fixed point $x^{*}$ in $M$. Also, $f$ is a Picard operator, that is, $\lim f^{n}(x)=x^{*}$ for all $x \in M$.

(see also $[9,10,19])$.

Theorem 2.2. [8](chap.4,31.1) Given a point $\left(t_{0}, x_{0}\right) \in \mathbb{R} \times \mathbb{R}^{n}$ consider a differential equation (1.1). Let $P$ be a Picard mapping that takes a function $\phi: t \rightarrow x$ to the function $P \phi: t \rightarrow x$ defined by

$$
(P \phi)(t)=x_{0}+\int_{t_{0}}^{t} v(\tau, \phi(\tau)) d \tau \quad \tau \in \mathbb{R}
$$

Note that $(P \phi)\left(t_{0}\right)=x_{0}$ for any $\phi$. The mapping $\phi: I \rightarrow \mathbb{R}^{n}$ is a solution to $\dot{x}=v(t, x)$ with the initial condition $\phi\left(t_{0}\right)=x_{0}$ if and only if $\phi=P \phi$.

Simply, the theorem states that the solution to a first-order differential equation is the "fixed point" of a Picard mapping. Theorem 2.1 gives us some conditions under which a mapping has one and only one fixed point. Thus, if we could construct a mapping that includes both types of functions in just the right way, we could take advantage of the existence and uniqueness of the fixed point of this mapping to prove the existence and uniqueness of the solution to our differential equation.

\section{Main results}

In this section, we are ready to state new and simple proofs of Theorems 1.1 and 1.2. To this end, we need some definitions.

Let $(M, \rho, \perp)$ be an orthogonal metric space $((M, \perp)$ is an O-set and $(M, \rho)$ is a metric space).

Definition 3.1. Let $\phi$ be a mapping of an open interval $I$ in $\mathbb{R}$ to $(M, \rho, \perp)$. The derivative of $\phi$ is defined by

$$
\dot{\phi}(t):=\lim _{s \rightarrow 0} \frac{\rho(\phi(t+s), \phi(t))}{s},
$$

where $t \in \mathbb{R}$ is a limit point of $I$ and $\phi(t) \perp \phi(t+s)$ if the limit exists. 
We now consider the tangent space to $(M, \rho, \perp)$ at a point.

Definition 3.2. Let $\phi$ be a differentiable mapping of an open interval $I$ in $\mathbb{R}$ to $(M, \rho, \perp)$. $\phi$ is said to leave the point $x$ for some $x \in M$ if $\phi(0)=x$. The derivative of $\phi$ at the point $t=0$ is a vector $v$ as:

$$
v=\dot{\phi}(0)=\left.\frac{d \phi}{d t}\right|_{t=0}
$$

The tangent space to $(M, \perp)$ at a point $x$ is the set of all vectors $v$ of all such curves leaving $x$ and denoted $T_{x} M$.

We turn our attention to the concept of the derivative of a mapping $f$ at a point.

Definition 3.3. Let $f: U \rightarrow V$ be a differentiable mapping from the subset $U$ of the orthogonal metric space $\left(M_{1}, \rho_{1}, \perp_{1}\right)$ into the subset $V$ of the orthogonal metric space $\left(M_{2}, \rho_{2}, \perp_{2}\right)$ and let $\phi: I \rightarrow U$ be a differentiable mapping which leaves the point $x \in U$ at $t=0$. The derivative of the mapping $f$ at the point $x$ is the mapping

$$
f_{* x}: T_{x} U \rightarrow T_{f(x)} V
$$

which carries the vector $v$ leaving the point $x$ of the curve $\phi$ into the vector $f_{* x}(v)$ leaving the point $f(x)$ of the curve $f(\phi)$ i.e.

$$
f_{* x}(v)=f_{* x}\left(\left.\frac{d \phi}{d t}\right|_{t=0}\right)=\left.\frac{d f(\phi)}{d t}\right|_{t=0} .
$$

Then we have the following result.

Proposition 3.1. Let $f: U \rightarrow \mathbb{R}^{n}$ be a smooth mapping $\left(f \in C^{r}, r \geq 1\right)$ from $U \subseteq\left(\mathbb{R}^{m}, \perp_{1}\right)$ to $\left(\mathbb{R}^{n}, \perp_{2}\right)$ and $x \in U$. Then $f$ satisfies the Lipchitz condition on each convex compact subset $V$ of $U$ with the Lipchitz constant $L$ equal to the supremum of the derivative of $f$ on $V$ :

$$
L=\sup _{x \in V}\left|f_{* x}\right| .
$$

Proof. Take any two points $x, y \in V, x \perp_{1} y$ and join them together with a line segment

$$
z(t)=x+t(y-x) ; \quad 0 \leq t \leq 1 .
$$

Since $V$ is convex, $z(t) \in V ; \quad \forall t \in[0,1]$. Now, we have

$$
\int_{0}^{1} \frac{d}{d t}(f(z(t)) d t=f(z(1))-f(z(0))=f(y)-f(x),
$$

and

$$
\int_{0}^{1} \frac{d}{d t}\left(f(z(t)) d t=\left.\int_{0}^{1} \frac{d f}{d z}\right|_{z(t)} \frac{d z}{d t}(t) d t=\int_{0}^{1} f_{* z(t)}(y-x) d t\right.
$$


Examining the absolute magnitude of this integral, we find

$$
\begin{aligned}
\left|\int_{0}^{1} f_{* z(t)}(y-x) d t\right| & \leq \int_{0}^{1}\left|f_{* z(t)}(y-x)\right| d t \\
& \leq \int_{0}^{1}\left|f_{* z(t)}\right||y-x| d t \\
& \leq\left(\int_{0}^{1}\left|f_{* z(t)}\right| d t\right)|y-x| \\
& \leq\left(\int_{0}^{1} L d t\right)|y-x| \\
& =|L .1-L .0||y-x|=L|y-x|
\end{aligned}
$$

We have thus determined that for any two points $x, y \in V$,

$$
|f(y)-f(x)|=\left|\int_{0}^{1} f_{* z(\tau)}(y-x) d \tau\right| \leq L|y-x|,
$$

and hence $f$ satisfies the Lipchitz condition on $V$ with the constant $L$.

Remark 3.1. In the previous proposition, since $f \in C^{1}$ the mapping $f_{*}=\frac{d f}{d x}$ which takes a given $x$ and returns the mapping $f_{* x}$ is continuous. Since $V$ is compact $\left|f_{* x}\right|$ actually attains its maximum value $L$.

Now, we are interested in obtaining a mapping that satisfies the properties of Theorem 2.1 and the fixed point of this mapping is the solution to (1.1). In this way, we prove the existence and uniqueness (Theorems 1.1 and 1.2) of the solution to $(1.1)$.

Because $v$ is differentiable at the point $\left(t_{0}, x_{0}\right) \in U$, there exists some neighborhood $C$ around $\left(t_{0}, x_{0}\right)$ such that $C \subset U$. Then there exist small enough numbers $a$ and $b$ such that

$$
C=\left\{(t, x) ;\left|t-t_{0}\right| \leq a,\left|x-x_{0}\right| \leq b\right\} \subset U \text {. }
$$

Clearly, $C$ is compact and $|v|$ attains its supremum over $C$. Similarly, $\left|v_{*}\right|=\left|\frac{d v}{d x}\right|$ attains its supremum over $C$. Let

$$
c=\sup _{C}|v|, \quad L=\sup _{C}\left|v_{*}\right| .
$$

We are interested in obtaining a function based on $v$, satisfying Lipchitz condition on each convex compact subset of $U$, including $C$ with the Lipchitz constant $L$. Let us separate $C$ into some subregions. There exists

$$
\dot{a}=\min \left\{a, \frac{b}{2 c}, \frac{1}{2 L}\right\}
$$

such that

$$
K_{0}=\left\{(t, x) ;\left|t-t_{0}\right| \leq \dot{a},\left|x-x_{0}\right| \leq c\left|t-t_{0}\right|\right\}
$$


lies in $C$.

For $\hat{b}=\frac{b}{2}$ and $\dot{x}$ with $\left|\dot{x}-x_{0}\right| \leq \dot{b}$ another point $\left(t_{0}, \dot{x}\right)$ can be considered such that

$$
K_{\grave{x}}=\left\{(t, x) ;\left|t-t_{0}\right| \leq \dot{a},|x-\dot{x}| \leq c\left|t-t_{0}\right|\right\} .
$$

The following argument shows that $a$ exists and is equal to $\min \left\{a, \frac{b}{2 c}, \frac{1}{2 L}\right\}$. Since $\left|x-x_{0}\right| \leq c\left|t-t_{0}\right| \leq c a ́$ then $a^{\prime}=\min \left\{a, \frac{b}{c}\right\}$ exists. On the other hand, by using triangle inequality, we find

$$
\left|x-x_{0}\right| \leq|x-\dot{x}|+\left|\dot{x}-x_{0}\right| \leq c a ́+\dot{b}=b .
$$

So, let $\dot{a}^{\prime}=\min \left\{a, \frac{b}{2 c}\right\}$. '́ will need one more bound later on, namely, the condition $\dot{a}<\frac{1}{L}$ (we are ignoring the trivial case $L=0$ ). So, let us go ahead and put $\dot{a}=\min \left\{a, \frac{b}{2 c}, \frac{1}{2 L}\right\}$.

We are trying to obtain the solution $\phi_{\dot{x}}: \mathbb{R} \rightarrow \mathbb{R}^{n}$ of (1.1) with the initial condition $\phi_{\dot{x}}\left(t_{0}\right)=\dot{x}$ expressed in the form $\phi_{\dot{x}}(t)=\dot{x}+h(t, \dot{x})$, though we can now remove the prime on $x$ :

$$
\phi_{x}(t)=x+h(t, x) .
$$

Then the mapping

$$
\phi:\left\{(t, x) ;\left|t-t_{0}\right| \leq \dot{a},\left|x-x_{0}\right| \leq \dot{b}\right\} \rightarrow \mathbb{R}^{n},
$$

defined by

$$
\phi(t, x)=\phi_{x}(t)
$$

is the "general" solution of (1.1).

One may easily verify the following lemma:

Lemma 3.1. For any solution $\phi_{x}$, the point $\left(t, \phi_{x}(t)\right)$ lies within $K_{x}$ for all $t$ such that $\left|t-t_{0}\right| \leq a ́$.

Recall that we are interested in obtaining a mapping that satisfies the properties of Theorem 2.1 and the fixed point of this mapping is the solution to (1.1). Let us first define the orthogonal metric space we will use. This space should include all the mappings which could possibly be solutions. Given some central initial condition $\left(t_{0}, x_{0}\right)$, the mapping $\phi$ should take the point $(t, x)$ from the region $\left|t-t_{0}\right| \leq$ á, $\left|x-x_{0}\right| \leq \dot{b}$ to $\mathbb{R}^{n}$.

Since $\phi_{x}$ must be a differentiable function in order to be a solution, it must be continuous on the set over which it is a solution. The space of all continuous functions $h(t, x)$ which added to $x$ could give us a solution $\phi_{x}$ with the initial condition $\phi_{x}\left(t_{0}\right)=x$ will be considered. Denote this space by $M$. Since $\phi$ takes the point $(t, x)$ from the region $\left|t-t_{0}\right| \leq a ́ a,\left|x-x_{0}\right| \leq \dot{b}$ to $\mathbb{R}^{n}$, the map $h$ must be over this region.

$$
h:\left\{(t, x) ;\left|t-t_{0}\right| \leq \dot{a},\left|x-x_{0}\right| \leq \dot{b}\right\} \rightarrow \mathbb{R}^{n} .
$$


Note that $h\left(t_{0}, x\right)=0$ for any $h \in M, x \in C$, where 0 is the zero vector in $\mathbb{R}^{n}$. In the space $M$, we can define a relation $\perp$ by

$$
h_{1} \perp h_{2} \Longleftrightarrow\left\|h_{1}\right\|\left\|h_{2}\right\| \leq c\left|t-t_{0}\right|\left(\left\|h_{1}\right\| \vee\left\|h_{2}\right\|\right),
$$

which is an orthogonality relation on $M$. It shows that the space $M$ is an orthogonal space.

Let $\rho: M \times M \rightarrow \mathbb{R}_{+}$be given by

$$
\rho\left(h_{1}, h_{2}\right)=\left\|h_{1}-h_{2}\right\|=\sup \left|h_{1}(t, x)-h_{2}(t, x)\right|,
$$

for all $h_{1}, h_{2} \in M$. Then $\rho$ is a metric on $M$ and the orthogonal metric space $M$ will be denoted by $(M, \rho, \perp)$. Since every $h$ is a continuous function over a closed and bounded subset of the Euclidean space, this supremum is actually attained. Hence, the orthogonal metric space $(M, \rho, \perp)$ is complete.

In the orthogonal metric space $(M, \rho, \perp)$, a mapping $A:(M, \rho, \perp) \rightarrow(M, \rho, \perp)$ can be defined by

$$
(A h)(t, x)=\int_{t_{0}}^{t} v(\tau, x+h(\tau, x)) d \tau,
$$

for $\left|t-t_{0}\right| \leq \dot{a},\left|x-x_{0}\right| \leq \dot{b}$. Clearly, $(\tau, x+h(\tau, x))$ is in the domain of $v$ for any $(\tau, x)$ in the appropriate region but we should be careful to check that $A h$ is in fact an element of $(M, \rho, \perp)$.

Lemma 3.2. For all $h \in M$, Ah $\in$.

Proof. Take any $h \in M$. By construction $A h$ is a function that satisfies (3.11). The function $h$ is continuous for any $(\tau, x)$ in its domain, so the point $(\tau, x+h(\tau, x))$ varies continuously with $(\tau, x)$ and since $v$ is also continuous on its domain $v$ varies continuously with $(\tau, x)$ as well. Taking the integral will then result in a continuous function of the boundary terms taken at $(t, x)$ and $\left(t_{0}, x\right)$. Thus, $A h$ is a continuous function of $(t, x)$ meaning $A h \in M$.

We now discuss some properties of mapping $A$.

1. $A$ is $\perp$-preserving mapping.

2. $A$ is $\perp$-contraction mapping.

3. $A$ is $\perp$-continuous mapping.

Proof. 1. We recall that $A$ is $\perp$-preserving, if for $h_{1}, h_{2} \in M, h_{1} \perp h_{2}$, we have $A h_{1} \perp A h_{2}$.

$$
\left|\left(A h_{1}\right)(t, x)\right|=\left|\int_{t_{0}}^{t} v\left(\tau, x+h_{1}(\tau, x)\right) d \tau\right|
$$




$$
\begin{aligned}
& \leq \int_{t_{0}}^{t}\left|v\left(\tau, x+h_{1}(\tau, x)\right)\right| d \tau \\
& \leq \int_{t_{0}}^{t} c d \tau \\
& =\left|c . t-c . t_{0}\right|=c\left|t-t_{0}\right| .
\end{aligned}
$$

So,

$$
\left\|A h_{1}\right\|\left\|A h_{2}\right\| \leq c\left|t-t_{0}\right|\left\|A h_{2}\right\| .
$$

Meaning that $A h_{1} \perp A h_{2}$.

2. We need to prove that for any $h_{1}, h_{2} \in M, h_{1} \perp h_{2},\left\|A h_{1}-A h_{2}\right\| \leq \lambda\left\|h_{1}-h_{2}\right\|$ for some constant $0<\lambda<1$. Let us then construct the mapping $A h_{1}-A h_{2}$.

$$
\begin{gathered}
\left.\left|\left(A h_{1}\right)(t, x)\right|=\left|\int_{t_{0}}^{t} v\left(\tau, x+h_{1}(\tau, x)\right) d \tau\right| \quad \text { (abbreviated } \int_{t_{0}}^{t} v_{1} d \tau\right), \\
\left(A h_{1}-A h_{2}\right)(t, x)=\int_{t_{0}}^{t} v_{1} d \tau-\int_{t_{0}}^{t} v_{2} d \tau=\int_{t_{0}}^{t}\left(v_{1}-v_{2}\right) d \tau .
\end{gathered}
$$

For a fixed $(\tau, x), v$ will act as a mapping that takes $h_{i}(\tau, x)$ to $v\left(\tau, x+h_{i}(\tau, x)\right)$. As $v$ was assumed to be continuously differentiable over its domain, we invoke Proposition 3.1 to find that $v$ satisfies the Lipchitz condition on each convex compact subset of its domain and therefore on each subset $C$ of $U$. Proposition 3.1 also gives us the Lipchitz constant $L(\tau)=\sup _{\left|x-x_{0}\right| \leq b}\left|v_{*}\right|$ where we have emphasized the fact that this $L$ depends on the choice of the constant $\tau$. Thus, for all points $(\tau, x)$,

$$
\left|v_{1}(\tau, x)-v_{2}(\tau, x)\right| \leq L(\tau)\left\|h_{1}-h_{2}\right\| .
$$

As seen earlier, the magnitude of any mapping in $M$ attains its supremum at some point in its domain, so we have

$$
\left\|A h_{1}-A h_{2}\right\|=\sup \left|A h_{1}(t, x)-A h_{2}(t, x)\right|=\left|A h_{1}\left(t_{m}, x_{m}\right)-A h_{2}\left(t_{m}, x_{m}\right)\right|,
$$

for some $\left(t_{m}, x_{m}\right) \in C$. Therefore,

$$
\begin{aligned}
\left\|A h_{1}-A h_{2}\right\| & =\left|\int_{t_{0}}^{t_{m}}\left(v_{1}\left(\tau, x_{m}\right)-v_{2}\left(\tau, x_{m}\right)\right) d \tau\right| \\
& \leq \int_{t_{0}}^{t_{m}}\left|\left(v_{1}\left(\tau, x_{m}\right)-v_{2}\left(\tau, x_{m}\right)\right)\right| d \tau \\
& \leq \int_{t_{0}}^{t_{m}} L(\tau)\left\|h_{1}-h_{2}\right\| d \tau \\
& =\int_{t_{0}}^{t_{m}} L(\tau) d \tau\left\|h_{1}-h_{2}\right\| .
\end{aligned}
$$


In $(3.5), L$ (without the parenthetical $\tau$ ) was designated the supremum of $\left|v_{*}\right|$ over all of $C$ i.e. over both the $t$ and $x$ domains meaning that

$$
\begin{aligned}
\left\|A h_{1}-A h_{2}\right\| & \leq \int_{t_{0}}^{t_{m}} L(\tau) d \tau\left\|h_{1}-h_{2}\right\| \\
& \leq \int_{t_{0}}^{t_{m}} L d \tau\left\|h_{1}-h_{2}\right\| \\
& =L\left|t_{m}-t_{0}\right|\left\|h_{1}-h_{2}\right\| \\
& \leq L a ́\left\|h_{1}-h_{2}\right\| .
\end{aligned}
$$

Lastly, we take advantage of the extra bound we placed on $a$ to find that $L a ́ \leq L \frac{1}{2 L}=\frac{1}{2}<1$. Thus, for all $h_{1}, h_{2} \in M, h_{1} \perp h_{2}$,

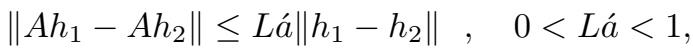

making $A$ a $\perp$-contraction mapping.

3. Suppose $\left\{h_{n}\right\}$ is an O-sequence in $M$ such that $\left\{h_{n}\right\}$ converging to $h \in M$. Because $A$ is $\perp$-preserving, $\left\{A h_{n}\right\}$ is an $\mathrm{O}$-sequence. For each $n \in \mathbb{N}$, since $A$ is $\perp$-contraction, we have

$$
\left\|A h_{n}(t, x)-A h(t, x)\right\| \leq L a ́\left\|h_{n}-h\right\| .
$$

As $n$ goes to infinity, it follows that $A$ is $\perp$-continuous.

The mapping $A$ defined above is $\perp$-preserving, $\perp$-contraction and $\perp$-continuous mapping over an orthogonal metric space $(M, \rho, \perp)$. The mapping $A$ satisfies the hypotheses of Theorem 2.1. Thus, the existence and uniqueness of its fixed point $h_{0} \in M$ is guaranteed by Theorem 2.1. The purpose of the present paper is to incorporate this in a Picard mapping of potential solutions to (1.1). Using the existence and uniqueness of $h_{0}$ to confirm the existence and uniqueness of the fixed point of the Picard mapping, which will in turn prove our main theorems.

First, recall that we are looking for solutions expressed in the form $\phi_{x}(t)=x+$ $h(t, x)$. If $h$ is a fixed point of $A$, then $\phi_{x}(t)=x+A h(t, x)$ and when the solution $\phi_{x}$ is the fixed point, our Picard mapping $\phi_{x}(t)$ will equal $\left(P \phi_{x}\right)(t)$. Hence,

$$
\begin{aligned}
\left(P \phi_{x}\right)(t) & =x+(A h)(t, x) \\
& =x+\int_{t_{0}}^{t} v(\tau, x+h(\tau, x)) d \tau \\
& =x+\int_{t_{0}}^{t} v\left(\tau, \phi_{x}(\tau)\right) d \tau
\end{aligned}
$$

By Theorem $(2.2), \phi_{x}$ is a solution to $\dot{x}=v(t, x)$ with $\phi_{x}\left(t_{0}\right)=x$ if and only if $\phi_{x}=P \phi_{x}$. We can now conclude this section with a new proof of the forthcoming results concerning the existence and uniqueness of the solution to (1.1) satisfying any initial condition in the domain of $v$. 
Theorem 3.1. (The Existence Theorem) Suppose the right-hand side $v$ of the differential equation $\dot{x}(t)=v(t, x)$ is continuously differentiable in a neighborhood of the point $\left(t_{0}, x_{0}\right) \in \mathbb{R} \times \mathbb{R}^{n}$. Then there exists a neighborhood of the point $t_{0}$ such that the solution to the differential equation is defined in this neighborhood with the initial condition $\phi\left(t_{0}\right)=x_{0}$ where $x$ is any point sufficiently close to $x_{0}$. Moreover, this solution depends continuously on the initial point $x$.

Proof. Given $v(t, x)$ as well as $\left(t_{0}, x_{0}\right)$, demarcate a neighborhood $C$ around the central point and use it to define the constants $a, b$; also, construct the orthogonal metric space $(M, \perp, \rho), \perp$-preserving, $\perp$-continuous, $\perp$-contraction mapping $A$ and a Picard mapping $P$ as determined by $v, C$ and the central point $\left(t_{0}, x_{0}\right)$. Since $M$ is an orthogonal complete metric space, the fixed point $h_{0}$ of $A$ must exist by Theorem 2.1. The function $g: \mathbb{R} \times \mathbb{R}^{n} \rightarrow \mathbb{R}^{n}$ given by

$$
g(t, x)=x+h_{0}(t, x),
$$

is therefore always well-defined in a neighborhood of $\left(t_{0}, x_{0}\right)$. Applying the Picard mapping

$$
(P g)(t, x)=x+\left(A h_{0}\right)(t, x)=x+h_{0}(t, x)=g(t, x),
$$

which proves that, by Theorem $2.2, g$ is the solution to the differential equation which satisfies the initial condition $g\left(t_{0}, x\right)=x$. The function which returns the value $x$ is continuous on $\mathbb{R} \times \mathbb{R}^{n}, h_{0}$ is continuous by construction and the sum of any two continuous function is continuous over the same domain. So $g$, the function of $t$ and $x$, is continuous over its domain. Thus, the solution depends continuously on the initial point $x$.

Uniqueness immediately follows:

Theorem 3.2. (The Uniqueness Theorem) Given the above conditions, there is only one possible solution for any given initial point, in the sense that all possible solutions are equal in the neighborhood under consideration.

Proof. Construct a neighborhood and mapping as above but now set $\dot{b}=0$, which restricts the initial $x$ under our consideration to the specific point $x_{0}$. Find the solution $g\left(t, x_{0}\right)=x_{0}+h_{0}\left(t, x_{0}\right)$. The uniqueness of the fixed point $h_{0}$ guarantees that this is the only solution with the initial condition $x_{0}$ that can be expressed in the form $x+h(t, x)$.

Now, consider any solution $\phi_{x_{0}}$ with $\phi_{x_{0}}\left(t_{0}\right)=x_{0}$. By Lemma 3.1, $\phi_{x_{0}}(t) \in K_{0}$ for all $t$ in our neighborhood. Label $\phi_{x_{0}}(t)-x_{0}$ by $h_{\phi}\left(t, x_{0}\right)$. This new function also clearly satisfies (3.11) and, furthermore, since any solution $\phi$ must be continuous, $h_{\phi}$ is also continuous. So, $h_{\phi} \in M$ and $\phi_{x_{0}}(t)=x_{0}+h_{\phi}\left(t, x_{0}\right)$. The uniqueness of $h_{0}$ shows that all possible solutions to the differential equation with a given initial condition are expressed in the form $\phi_{x_{0}}=x_{0}+h\left(t, x_{0}\right)$ for $h \in M$. As there is only one such function possible, the solution $g$ is thus unique. 


\section{R E F E R E N C E S}

1. R. P. Agarwal, M. Benchohra and S. Hamani: Boundary value problems for fractional differential equations. Georgian Math. J. 163 (2009) 401-411.

2. R. P. Agarwal, D. O'Regan and S. Stanek: Positive solutions for Dirichlet problems of singular nonlinear fractional differential equations. J. Math. Anal. Appl. 371 (2010) 57-68.

3. R. P. Agarwal, M. Benchohra and S. Hamani: A survey on existence results for boundary value problems of nonlinear fractional differential equations and inclusions. Acta Appl. Math. 109 (2010) 973-1033.

4. R. P. Agarwal, D. Franco and D. O'Regan: Singular boundary value problems for first and second order impulsive differential equations. Aequat. Math. 69 (2005) 83-96.

5. B. Ahmad and J. J. Nieto: Boundary Value Problems for a Class of Sequential Integro differential Equations of Fractional Order. J. Func. Space. Appl. (2013) Article ID 149659.

6. A. Amini-Harandi and H. Emami: A fixed point theorem for contraction type maps in partially ordered metric spaces and application to ordinary differential equations. Nonlinear Anal. 72 (2010) 2238-2242.

7. V. I. Arnold: Ordinary Differential Equations. Translated and Edited by Richard A. Silverman, The M. I. T. Press, 1998.

8. V. I. Arnold: Ordinary Differential Equations, Translated from the Russian by Roger Cooke, Springer-verlog, 1992.

9. H. Baghani and M. Ramezani: Contractive gauge functions in strongly orthogonal metric spaces. Int. J. Nonlinear Anal. Appl. Article in press ISSN: 2008-6822 (electronic).

10. H. Baghani, M. Eshaghi Gordji and M. Ramezani: Orthogonal sets: their relation to the axiom of choice and a generalized fixed point theorem. J. Fixed Point Theory Appl. 183 (2016) 465-477.

11. S. BANACH: Sur les operations dans les ensembles abstraits et leur application aux equations integrales. Fund. Math. 3 (1922) 133-181.

12. M. Belmekki, J. J. Nieto and R. Rodriguez-Lopez: Existence of solution to a periodic boundary value problem for a nonlinear impulsive fractional differential equation. E. J. Qual. Theory Diff. Equ. 16 (2014) 1-27.

13. M. Eshaghi Gordji, M. Ramezani, M. De La Sen and Y. J. Cho: On orthogonal sets and Banach fixed point theorem. Fixed Point Theory. 182 (2017) $569-578$.

14. A. A. Ivanov: Fixed point theory. J. Sovi. Math. 12 (1979) 1-64.

15. E. Karapinar and R. P. Agarwal: A note on 'Coupled fixed point theorems for $\alpha-\psi$-contractive-type mappings in partially ordered metric spaces. Fixed Point Theory Appl. 16 (2013) 2013:216.

16. J. J. Nieto, R. L. Pouso and R. Rodrguez-Lopez: Fixed point theorems in ordered abstract sets. Proc. Amer. Math. Soc. 135 (2007) 2505-2517.

17. J. J. Nieto, R. L. Pouso and R. Rodrguez-Lopez: Contractive mapping theorems in partially ordere sets and applications to ordinary differential equations. Order 22 (2005) 223-239. 
18. J. J. Nieto, R. L. Pouso and R. Rodrguez-Lopez: Existence and uniqueness of fixed point in partially ordered sets and applications to ordinary differential equations. Acta Math. Sin. 23 (2007) 2205-2212.

19. M. Ramezani: Orthogonal metric space and convex contractions. Int. J. Nonlinear Anal. Appl. 62 (2015) 127-132.

Madjid Eshaghi Gordji

Department of Mathematics, Semnan University

P. O. Box 35195-363

Semnan, Iran

meshaghi@semnan.ac.ir

Hasti Habibi

Department of Mathematics, Semnan University

Semnan, Iran

hastihabibi1363@gmail.com 